\title{
Modified Technique of Pancreaticogastrostomy for Soft Pancreas with Two Continuous Hemstitch Sutures: A Single-Center Prospective Study
}

\author{
Feng Zhu $\cdot$ Min Wang $\cdot$ Xin Wang $\cdot$ Rui Tian • \\ Chengjian Shi • Meng Xu • Ming Shen • Juan Han • \\ Ninanian Luo $\cdot$ Renyi Qin
}

Received: 27 August 2012 / Accepted: 5 March 2013 / Published online: 19 March 2013

(C) 2013 The Author(s). This article is published with open access at Springerlink.com

\begin{abstract}
Postoperative pancreatic fistula (POPF) remains a persistent problem after pancreaticoduodenectomy (PD), especially in the presence of a soft, nonfibrotic pancreas. To reduce the risk of POPF, pancreaticogastrostomy (PG) is an optional reconstruction technique for surgeons after PD. This study presents a new technique of PG for a soft, nonfibrotic pancreas with double-binding continuous hemstitch sutures and evaluates its safety and reliability. From January 2011 to June 2012, 92 cases of patients with periampullary malignancy with a soft pancreas underwent this technique. A modified technique of PG was performed with two continuous hemstitch sutures placed in the mucosal and seromuscular layers of the posterior gastric wall, respectively. Then the morbidity and mortality was calculated. This technique was applied in 92 patients after PD all with soft pancreas. The median time for the anastomosis was 12 min (range, 8-24). Operative mortality was zero, and morbidity was $16.3 \%(n=15)$, including hemorrhage $(n=2)$, biliary fistula $(n=2)$, pulmonary infection $(n=1)$, delayed gastric emptying (DGE; $n=5,5.4 \%$ ), abdominal abscess ( $n=3$, one caused by PF), and POPF $(n=2,2.2 \%)$. Two patients developed a pancreatic fistula (one type A and one type B) classified according to the International Study Group on Pancreatic Fistula. The described technique is a simple and safe reconstruction procedure after PD, especially for patients with a soft and fragile pancreas.
\end{abstract}

Keywords Periampullary carcinoma .

Pancreaticogastrostomy · Pancreaticoduodenectomy ·

Postoperative pancreatic fistula $\cdot$ Continuous

hemstitch sutures

$\overline{\text { Feng Zhu, MD, and Min Wang, MD, contributed equally to this work. }}$

Electronic supplementary material The online version of this article (doi:10.1007/s11605-013-2183-8) contains supplementary material, which is available to authorized users.

F. Zhu $\cdot$ M. Wang $\cdot$ X. Wang $\cdot$ R. Tian $\cdot$ C. Shi $\cdot$ M. Xu $\cdot$ M. Shen $\cdot$

J. Han $\cdot$ N. Luo $\cdot$ R. Qin $(\triangle)$

Department of Biliary-Pancreatic Surgery, Affiliated Tongji

Hospital, Tongji Medical College, Huazhong University

of Science and Technology, 1095 Jiefang Ave,

Wuhan City, Hubei Province 430030, People's Republic of China

e-mail: ryqin@tjh.tjmu.edu.cn

\section{Introduction}

The incidence of postoperative complications remains high after pancreaticoduodenectomy (PD), especially in patients with "soft" pancreatic tissue remnants. ${ }^{1,2}$ The formation of POPF has a major influence on the postoperative course, possibly leading to life-threatening complications such as postoperative pancreatic hemorrhage (PPH) and/or peritonitis. ${ }^{3,4}$ Risk factors for pancreatic fistula include a soft pancreas, a small pancreatic duct, underlying pathology, and the surgeon's experience. ${ }^{5}$ Many technical modifications have been suggested for pancreaticoenteric anastomosis to decrease pancreatic leakage rate. ${ }^{6-8}$ However, no "gold standard" surgical technique has been established.

The most common methods of pancreatic anastomosis include pancreaticojejunostomy (PJ) and pancreaticogastrostomy (PG). At present, there is still no consensus on the choice of 
anastomotic technique (PJ vs. PG). ${ }^{9} 10$ In one recent RCT, the pancreatic fistula rate was significantly lower after PG (4\%) compared with PJ (18\%). However, in other prospective randomized trials, there was no statistical difference between them with regard to outcomes. ${ }^{9,11-13}$ Although these trials showed no difference regarding pancreatic fistula or overall complication rates compared with PJ, PG is still an option for reconstruction with some theoretical advantages over PJ. ${ }^{14}$

Several PG anastomotic techniques have been reported, including mattress sutures, the so-called binding PG using two purse-string sutures, or one binding purse-string and two transfixing mattress sutures. ${ }^{15,16}$ The aim of this prospective, noncomparative study was to modify and assess PG in a single center in 92 consecutive cases. The preliminary results are encouraging and showed that this technique could be performed safely, simply, and with a low pancreatic fistula rate for cases of soft pancreas.

\section{Patients and Methods}

Ninety-two consecutive patients with resectable periampullary tumors undergoing PD with PG at the Institute of BiliaryPancreatic Surgery, Tongji Hospital, Tongji Medical College, Huazhong Scientific and Technological University, China, were recruited from January 2011 to June 2012. Patients' data, including demographics (age, sex, and diagnosis), complications, hospital mortality, hospital stay, perioperative parameters (operating time, blood loss, and blood transfusion), postoperative interventional procedures, or reoperations was prospectively recorded. According to preoperative measurement of the diameter of the pancreatic duct using CT films (nondilated pancreatic duct), intraoperative findings of the texture of the remnant pancreas (pancreatic body or tail), and postoperative pathological findings of the pancreatic cut margins, all patients who underwent the operation had a soft, nonfibrotic pancreas. Patients received octreotide (600 $\mu \mathrm{g} / \mathrm{day})$ until postoperative day 5. A nasogastric tube was left in place until postoperative day 5 to protect the PG. Postoperative pancreatic fistula (POPF) was defined and classified according to the International Study Group on Pancreatic Fistula definition, as more than three times the serum concentration in drainage fluid on or after postoperative day $3 .^{17}$ Associated clinical findings including biliary fistula, delayed gastric emptying (DGE), and operative mortality were defined and classified according to the International Study Group on Pancreatic Surgery. ${ }^{1}$ A biliary fistula was defined as persistence of biliary drainage for more than 5 days, confirmed by fistulography. Gastric emptying was considered delayed when postoperative gastric suction was required for more than 10 days or when the patient was not able to tolerate a solid diet on or before postoperative day 14 . Operative mortality was any death occurring within 30 days of the procedure.
Pancreaticogastrostomy Procedure

PD was performed without pylorus-preserving procedures by a single group of surgeons. The extent of lymph node dissection in our procedures includes stations $1,3,4,5,6,7$, 8, 9, 12 (12h, A, p, b, and c), 13, 14, 15, and 16A2,

The pancreas was transected with at least a 2 -cm surgical margin from the tumor using an electrotome or ultrasound knife. After the surgical specimen was delivered, PG anastomosis was performed according to our technique.

\section{Preparation of Pancreatic Stump}

Any bleeding from the cut surface of the pancreatic stump was stopped using electrical coagulation or absorbable sutures (4-0 PDS-II). The remnant of the pancreas was dissected from the splenic vein and the surrounding tissues for a distance of approximately $2 \mathrm{~cm}$ from its cut edge. Several small veins running between the pancreas and the splenic vein were carefully ligated and divided. After adequate isolation when the isolated pancreatic remnant was pulled forward, the splenic artery and splenic vein could be seen and separated from a small area of the pancreas site to be pulled into the stomach. The main pancreatic duct location in the pancreatic stump was then identified with probes and a plastic stent (2- to 3-mm diameter, a disposable nelaton catheter, Suzhou Riyuexing Plastic Co. Ltd, Suzhou, China) was inserted into the pancreatic duct for approximately 3$5 \mathrm{~cm}$ with approximately $3 \mathrm{~cm}$ left outside the duct (about eight centimeters in total length). Once the suture was tied, the stent was fixed to the pancreatic duct to avoid inadvertent pancreatic duct ligation (Fig. 1a).

\section{Preparation of Stomach}

A standard antrectomy, nonpylorus-preserving procedure, was performed at the level of the third or fourth transverse vein on the lesser curvature and at the confluence of the gastroepiploic veins on the greater curvature using a linear gastrointestinal stapler. The sealed distal gastric stump was held with an intestinal clamp or temporarily closed using a linear gastrointestinal stapler. A small section of the seromuscular layer of the posterior gastric wall was removed with the length of the incision based on the size of the remnant of the pancreas (approximately $2-3 \mathrm{~cm}$ in diameter). Until this point the mucosa of the posterior gastric wall was kept intact (Figs. 1a and 2a). The appropriate position of the incision was selected so that the pancreatic stump could enter this hole without tension. A seromuscular continuous hemstitch suture (3-0 prolene) was then preplaced, involving approximately $1 \mathrm{~cm}$ of the seromuscular gastric wall (Figs. 1b and 2a). A lateral stab incision of $3 \mathrm{~cm}$ was made in the mucosa of the posterior gastric wall under the area of the incised seromuscular layer 

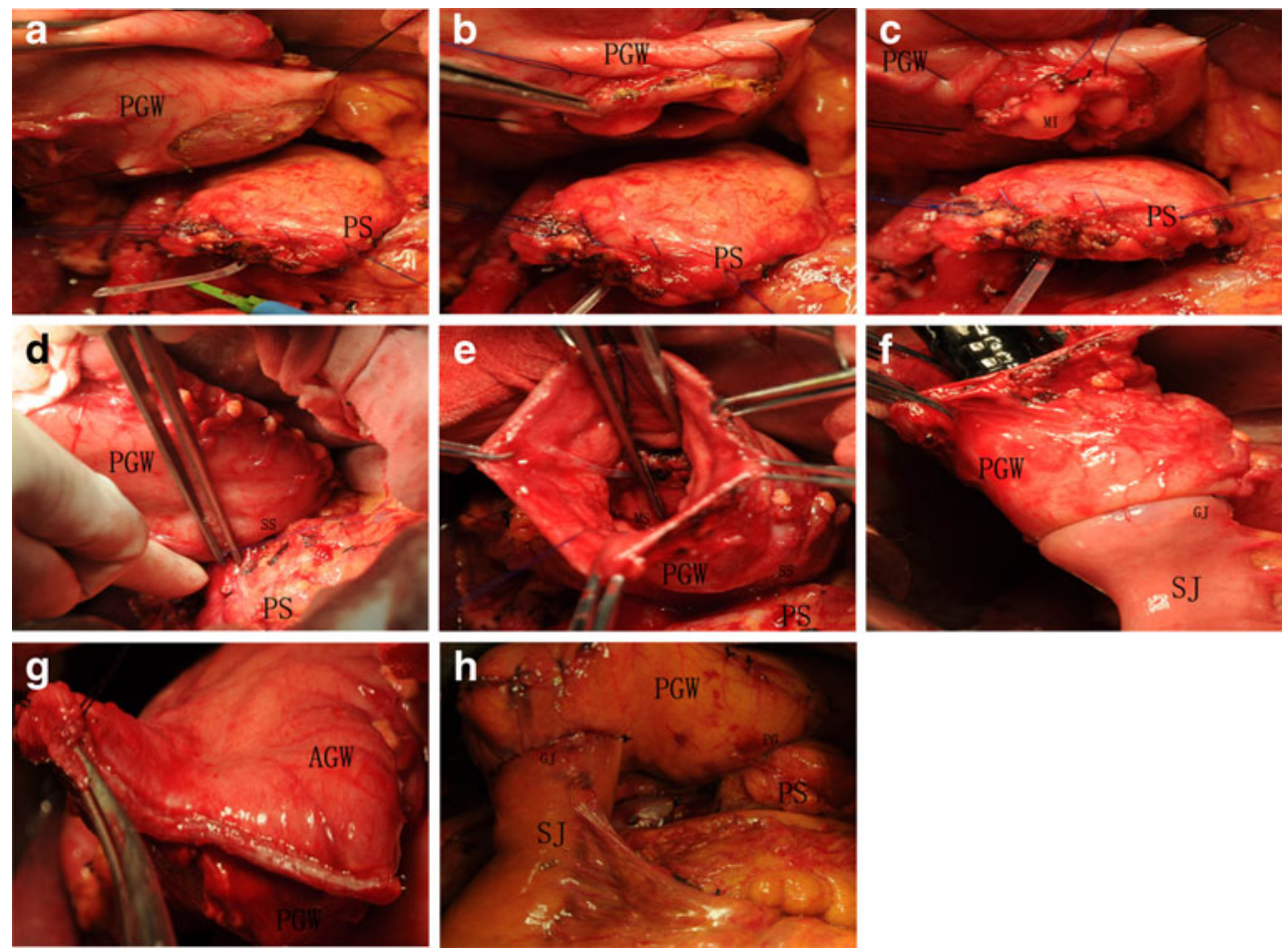

Fig. 1 Colored picture showed procedures of pancreaticogastrostomy. a The pancreatic stump (PS) were prepared, and a seromuscular incision is made in the posterior gastric wall $(P G W)$ exposing the gastric mucosa. b A seromuscular continuous hemstitch suture was preplaced in the seromuscular gastric wall and a lateral stab incision was made in the mucosal layer of the posterior gastric wall. c A continuous hemstitch suture was preplaced around the mucosal incision $(M I)$. $\mathbf{d}$ The pancreatic remnant was pulled into the stomach. The seromuscular continuous

and another continuous hemstitch suture (3-0 prolene) was preplaced around the mucosal layer incision, involving approximately $1 \mathrm{~cm}$ of the mucosa of the gastric wall (Fig. 1b, c).

\section{Pancreaticogastrostomy Anastomosis}

The sealed distal gastric stump was opened by moving the intestinal clamp or incising approximately $2 \mathrm{~cm}$ from the edge of the distal gastric stump that was closed by the linear gastrointestinal stapler. The preset suture placed in the mucosa of the posterior gastric wall was drawn into the gastric cavity through the opening of the distal gastric stump (Fig. 2b). The pancreatic remnant was then pulled with slight tension on the holding sutures through the opening in the posterior gastric wall into the stomach. This procedure was performed very gently to ensure tight wrapping of the posterior gastric wall around the pancreatic remnant and to avoid laceration of the pancreas. Ideally, the pancreatic remnant should protrude above the posterior gastric wall by $1 \mathrm{~cm}$. First, the seromuscular continuous hemstitch suture was tied at the lowest part of the pancreatic remnant (Figs. 1d and 2c). Then, through the hemstitch suture (seromuscular suture (SS)) was tied at the lowest part of the pancreatic remnant. e The preset suture placed in the mucosal layer of the posterior gastric wall (mucosal suture $(M S)$ ) was drawn into the gastric cavity and tied. f The gastrojejunostomy (GJ) was constructed in the posterior gastric wall. $\mathbf{g}$ the distal gastric stump was closed using another linear gastrointestinal stapler. $\mathbf{h}$ The pancreaticogastrostomy $(P G)$ and gastrojejunostomy were completed. $S J$ stump of the jejunum, $A G W$ anterior gastric wall

incision in the sealed distal gastric stump, the mucosal continuous hemstitch suture was tied approximately $1 \mathrm{~cm}$ off the margin of the pancreas (Figs. 1e and 2d). Each ligation should be carefully performed because the normal pancreas is soft and fragile. An end-to-side technique was used to construct a gastrojejunostomy using stapled anastomosis between the posterior gastric wall and the stump of the jejunum (Fig. 1f). The opening of the distal gastric stump was closed using another linear gastrointestinal stapler completing the pancreaticogastric anastomosis (Fig. 1g, h).

\section{Other Anastomoses}

Further reconstruction of digestive continuity was obtained by end-to-side hepaticojejunostomy, and side-to-side enteroenterostomy. A single soft silicone drain (Suzhou Riyuexing Plastic Co. Ltd, Suzhou, China) was inserted through a left-sided abdominal stab incision and placed in the vicinity of the pancreaticogastric anastomosis. An additional drain was placed on the right side for drainage of the hepaticojejunostomy. The drains were left in place at least 7 days after surgery and removed when less than $30 \mathrm{~mL}$ of clear fluid was drained per day. 
Fig. 2 a A seromuscular continuous hemstitch suture (seromuscular suture (SS)) was preplaced the posterior gastric wall $(P G W)$, involving approximately $1 \mathrm{~cm}$ of the seromuscular gastric wall. b The sealed distal gastric stump was opened and the preset suture placed in the mucosal layer of the posterior gastric wall $(M)$ was drawn into the gastric cavity through the opening in the distal gastric stump. c The

seromuscular continuous hemstitch suture (SS) was tied at the lowest part of the pancreatic stump (PS). d The mucosal continuous hemstitch suture (mucosal suture $(M S)$ ) was tied approximately $1 \mathrm{~cm}$ from the margin of the pancreas. $S$ spleen, $A G W$ anterior gastric wall
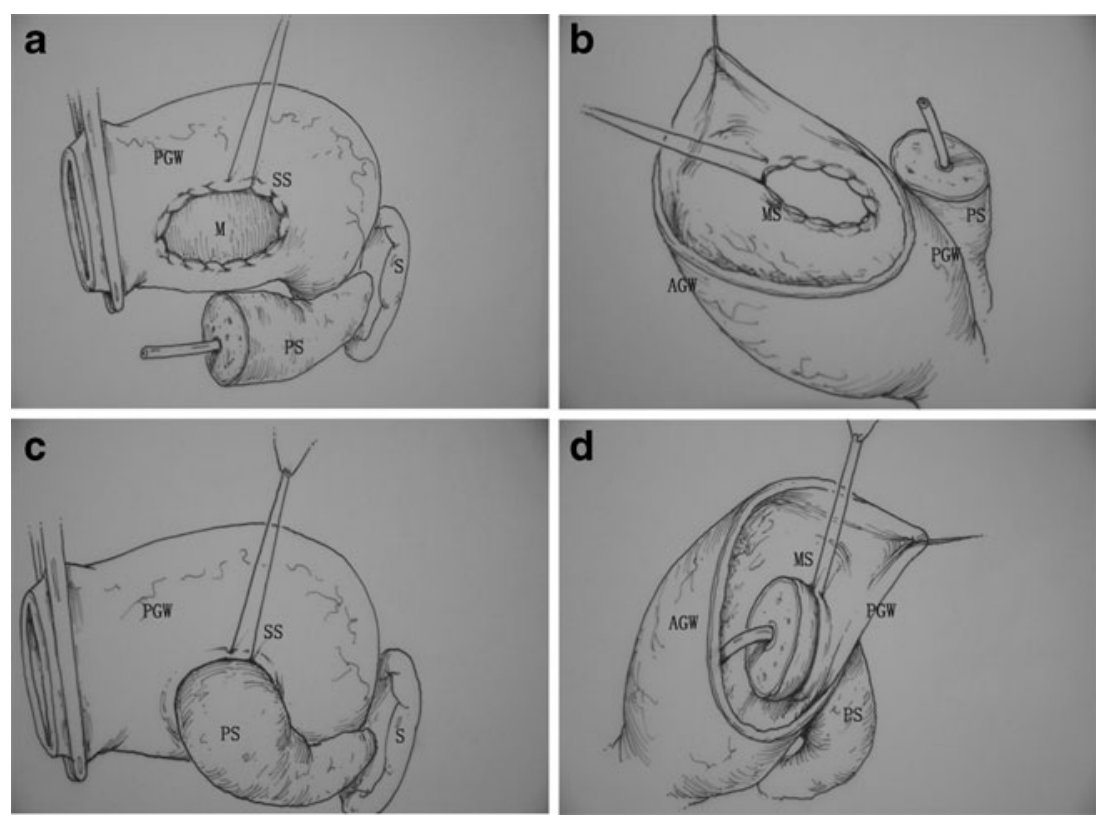

\section{Results}

A total of 92 patients underwent PD with the new modified PG technique. There were 43 men and 49 women with a median age of 53.7 (range, 33-72) years. The indications for PD were as follows: 18 pancreatic adenocarcinomas, 22 distal bile duct cancers, 27 ampulla of Vater cancers, 23 adenocarcinomas of the duodenum, and 2 intraductal papillary mucinous neoplasms. All patients had a soft and nonfibrotic pancreatic remnant identified by preoperative CT, intraoperative findings, and postoperative pathological examination of the pancreatic cut margins. The median blood loss was $650 \mathrm{~mL}$ (range, 320-1,300), 38 of 92 patients required transfusion and the median amount for 38 patients was $460 \mathrm{~mL}$ (range, 200-1,400). The median time for the PG (incision of the posterior gastric wall to finishing the mucosal continuous hemstitch suture) was $12 \mathrm{~min}$ (range, 8-24). The mean operation time was $4.1 \mathrm{~h}$ (range, 3.7-6.5) and the median hospital stay for the 92 patients was 15 days (range, 12-38).

There were no operative or hospital deaths or reoperations. Complications occurred in $15(16.3 \%)$ patients (Table 1), including hemorrhage $(n=2)$, biliary fistula $(n=2)$, pulmonary infection ( $n=1)$, DGE $(n=5,5.4 \%)$, abdominal abscess $(n=3$, one caused by PF), and POPF ( $n=2,2.2 \%)$. According to the international clinical grading system, one patient had grade A POPF. This case was treated conservatively and fed orally without additional intervention. One patient had grade B POPF and underwent an interventional drainage procedure with B-mode ultrasonic guidance. No patients had grade $\mathrm{C}$ POPF. The most frequent complication was DGE, which was treated with maintenance or reinsertion of a nasogastric tube, administration of erythromycin, and parenteral nutrition. Two patients had hemorrhage including one perianastomotic ulcer and one hemorrhage from the cut surface of the pancreatic stump which was treated by gastroscopy without reoperation. Abdominal abscess occurring in one case was caused by PF (grade B) and was treated with interventional drainage and another two cases underwent conservative treatment.

\section{Discussion}

Although pancreatoduodenectomy has become more standardized during recent decades, controversy still exists about the reconstruction of digestive continuity. The reconstructive procedures of the remnant pancreas are classified into PJ and PG. ${ }^{18-21}$ PG, first reported in 1946, has been reestablished as a secure method of anastomosis as an alternative to the standard PJ. To date, approximately 3,800 pancreaticogastrostomies have been reported. ${ }^{22} \mathrm{PG}$ has been used by several surgeons in recent years because of its theoretical advantages over PJ. Some authors have emphasized the texture of the pancreatic remnant as a predisposing factor of complications. Obviously, anastomotic dehiscence with subsequent leakage is more common in a soft pancreatic remnant, which usually occurs in patients without primary or subsequent pancreatitis. ${ }^{23}$ This can be explained by the inability of soft pancreatic tissue to hold sutures adequately. As a result, it is considered that PG should be performed for "soft pancreas" because a soft pancreas is commonly hypertrophied and it is easier to pull the pancreatic remnant into the stomach than into the jejunum. 
Table 1 Background characteristics, surgical outcomes, and postoperative complications

\begin{tabular}{|c|c|}
\hline \multicolumn{2}{|l|}{ Background characteristics } \\
\hline $\operatorname{Sex}(M / F)$ & $43 / 49$ \\
\hline Age (year) & $53.7(33-72)$ \\
\hline BMI & $27.4(16.7-42.5)$ \\
\hline Disease $(n)$ & 92 \\
\hline Pancreatic tumor & $18(20 \%)$ \\
\hline Cholangiocarcinoma & $22(24 \%)$ \\
\hline Ampullary tumor & $27(29 \%)$ \\
\hline Duodenal tumor & $23(25 \%)$ \\
\hline IPMN & $2(2 \%)$ \\
\hline \multicolumn{2}{|l|}{ Surgical outcomes } \\
\hline Operation time (h) & $4.1(3.7-6.5)$ \\
\hline Pancreaticojejunostomy time (h) & $12(8-24)$ \\
\hline Operative blood loss (mL) & $650(320-1,300)$ \\
\hline Required transfusion $(n)$ & $38(41.3 \%)$ \\
\hline Blood transfusion $(\mathrm{mL})$ & $460(200-1,400)$ \\
\hline Hospital stay (day) & $15(12-38)$ \\
\hline \multicolumn{2}{|l|}{ Postoperative complications $(n)$} \\
\hline Morbidity rate & $15(16.3 \%)$ \\
\hline Hemorrhage & 2 \\
\hline Delayed gastric emptying & $5(5.4 \%)$ \\
\hline Biliary fistula & 2 \\
\hline Pulmonary infection & 1 \\
\hline Abdominal abscess & 3 \\
\hline POPF & $2(2.2 \%)$ \\
\hline Grade A & 1 \\
\hline Grade B & 1 \\
\hline Grade C & 0 \\
\hline Re-operation & 0 \\
\hline Mortality & 0 \\
\hline
\end{tabular}

$B M I$ body mass index, IPMN intraductal papillary mucinous neoplasm, $P O P F$ postoperative pancreatic fistula

Recently, Ohigashi et al. and Peng et al. reported a new modified technique describing the theoretical advantages of the binding and transfixing modifications. ${ }^{14,15}$ Bartsch et al. reported another new technique for PG, which combines one binding purse-string and two transfixing mattress sutures between the pancreatic stump and the posterior gastric wall. Although Bartsch et al. modified the techniques of Ohigashi from 4 to 6 mattress sutures to two transfixing mattress sutures, these techniques still require transpancreas sutures. Every suture carries the risk for pancreatic laceration resulting in pancreatic leakage, especially in a fragile and soft pancreatic remnant. ${ }^{24}$ Peng et al. introduced four types of binding PG using binding purse-string without transpancreas sutures. Types I and II placed the suture in the mucosal layer through an opening in the gastric anterior wall or the sealed distal gastric stump. However, this technique was not comfortable for surgeons and prolonged the operation time. Types III and IV were designed to avoid opening the gastric anterior wall or the sealed distal gastric stump, and, instead, the binding anastomosis was performed behind the posterior stomach wall directly. However, it was also not an easy procedure nor was it safe to place the inner suture. ${ }^{16}$

In this study, we reported a new anastomosis technique using two continuous hemstitch sutures without transpancreas sutures for pancreatogastrostomy for soft pancreas. Compared with other anastomosis techniques, our method has the following advantages: (1) it is simple and easy. First, only two continuous hemstitch sutures are placed in the mucosal and seromuscular layers of the posterior gastric wall. Second, the mucosal layer of the posterior gastric wall is not removed and has only a lateral stab incision. In addition, the inner continuous hemstitch suture is preset out of the gastric cavity and drawn in through the opening of the sealed distal gastric stump. It is comfortable and easy for surgeons to suture and bind the pancreas in this way. Third, we use the opening of the sealed distal gastric stump and avoid excising the gastric anterior wall. The median time to perform PJ using this technique was only 12 min (range, 8-24). (2) It is safe. First, our modified technique can be carried out safely on the normal pancreas to avoid a PF because there is no suture through the pancreas. Second, a common reason for reoperation is upper gastrointestinal hemorrhage. Hemorrhage occurs most often at the site of the gastroenterostomy or PG. Bleeding following PG can occur from either the anastomotic site or the cut surface of the pancreas. The modified technique with continuous hemstitch sutures could effectively prevent hemorrhage in the cut edges of both the mucosal and seromuscular layers of the posterior gastric wall. Finally, the inner continuous hemstitch sutures are visibly tightened in the gastric cavity.

In conclusion, the described pancreatogastrostomy modification with double-binding continuous hemstitch sutures in the posterior gastric wall is simple, safe, and reliable. Because this work is preliminary, the technique must be evaluated in larger controlled trials.

Acknowledgments This study was funded by The National Natural Science Foundation of China (No. 81071775, 81101621, 81172064, and 81001068), National "Eleventh Five-Year" Scientific and Technological Support Projects (No. 2006BAI02A13-402), Key Projects of Science Foundation of Hubei Province (No. 2011CDA030), and Research Fund of Young Scholars for the Doctoral Program of Higher Education of China (No. 20110142120014).

Conflict of Interest The authors declare that they have no conflict of interest. 
Open Access This article is distributed under the terms of the Creative Commons Attribution License which permits any use, distribution, and reproduction in any medium, provided the original author(s) and the source are credited.

\section{References}

1. Butturini G, Marcucci S, Molinari E, Mascetta G, Landoni L, Crippa S, Bassi C: Complications after pancreaticoduodenectomy: the problem of current definitions. J Hepatobiliary Pancreat Surg 2006, 13:207-211.

2. DeOliveira ML, Winter JM, Schafer M, Cunningham SC, Cameron JL, Yeo CJ, Clavien PA: Assessment of complications after pancreatic surgery: A novel grading system applied to 633 patients undergoing pancreaticoduodenectomy. Ann Surg 2006, 244:931-937; discussion 937-939.

3. Callery MP, Pratt WB, Vollmer CM, Jr.: Prevention and management of pancreatic fistula. J Gastrointest Surg 2009, 13:163-173.

4. Lai EC, Lau SH, Lau WY: Measures to prevent pancreatic fistula after pancreatoduodenectomy: a comprehensive review. Arch Surg 2009, 144:1074-1080.

5. Lermite E, Pessaux P, Brehant O, Teyssedou C, Pelletier I, Etienne S, Arnaud JP: Risk factors of pancreatic fistula and delayed gastric emptying after pancreaticoduodenectomy with pancreaticogastrostomy. $J$ Am Coll Surg 2007, 204:588-596.

6. Govindarajan A, Tan JC, Baxter NN, Coburn NG, Law CH: Variations in surgical treatment and outcomes of patients with pancreatic cancer: a population-based study. Ann Surg Oncol 2008, 15:175-185.

7. Kim JH, Yoo BM, Kim WH: Which method should we select for pancreatic anastomosis after pancreaticoduodenectomy? World $J$ Surg 2009, 33:326-332.

8. Kleespies A, Albertsmeier M, Obeidat F, Seeliger H, Jauch KW, Bruns CJ: The challenge of pancreatic anastomosis. Langenbecks Arch Surg 2008, 393:459-471.

9. Bassi C, Falconi M, Molinari E, Salvia R, Butturini G, Sartori N, Mantovani W, Pederzoli P: Reconstruction by pancreaticojejunostomy versus pancreaticogastrostomy following pancreatectomy: results of a comparative study. Ann Surg 2005, 242:767-771, discussion 771-763.

10. Dixon E, Fingerhut A, Bassi C, Sutherland F, McKay A: Metaanalysis of pancreaticojejunostomy versus pancreaticogastrostomy reconstruction after pancreaticoduodenectomy: authors' comment (Br J Surg 2006; 93: 929-936). Br J Surg 2006, 93:1435.

11. Niedergethmann M, Dusch N, Widyaningsih R, Weiss C, Kienle P, Post S: Risk-adapted anastomosis for partial pancreaticoduodenectomy reduces the risk of pancreatic fistula: a pilot study. World J Surg 2010, 34:1579-1586.

12. Fernandez-Cruz L, Cosa R, Blanco L, Lopez-Boado MA, Astudillo E: Pancreatogastrostomy with gastric partition after pylorus-preserving pancreatoduodenectomy versus conventional pancreatojejunostomy: a prospective randomized study. Ann Surg 2008, 248:930-938.

13. Aranha GV, Hodul P, Golts E, Oh D, Pickleman J, Creech S: A comparison of pancreaticogastrostomy and pancreaticojejunostomy following pancreaticoduodenectomy. J Gastrointest Surg 2003, 7:672-682.

14. Ohigashi H, Ishikawa O, Eguchi H, Sasaki Y, Yamada T, Kishi K, Noura S, Takachi K, Miyashiro I, Oue M, et al: A simple and safe anastomosis in pancreaticogastrostomy using mattress sutures. $\mathrm{Am}$ J Surg 2008, 196:130-134.

15. Peng SY, Hong DF, Liu YB, Li JT, Tao F, Tan ZJ: A pancreas suture-less type II binding pancreaticogastrostomy. Zhonghua Wai Ke Za Zhi 2009, 47:1764-1766.

16. Peng SY WX, Liu Yingbin, HONG Defei, Li Jiangtao, Tao Feng, Tan Zhijian: Peng's pancreaticogastrostomy - comparative study on types I and II procedures (with types III and IV appended). Journal of Surgery Concepts \& Practice 2009, 14:5.

17. Bassi C, Dervenis C, Butturini G, Fingerhut A, Yeo C, Izbicki J, Neoptolemos J, Sarr M, Traverso W, Buchler M: Postoperative pancreatic fistula: an international study group (ISGPF) definition. Surgery 2005, 138:8-13.

18. Adams DB: The pancreatic anastomosis: the danger of a leak, which anastomotic technique is better? J Gastrointest Surg 2009, 13:1182-1183.

19. Bassi C, Falconi M, Molinari E, Mantovani W, Butturini G, Gumbs AA, Salvia R, Pederzoli P: Duct-to-mucosa versus endto-side pancreaticojejunostomy reconstruction after pancreaticoduodenectomy: results of a prospective randomized trial. Surgery 2003, 134:766-771.

20. Berger AC, Howard TJ, Kennedy EP, Sauter PK, Bower-Cherry M, Dutkevitch S, Hyslop T, Schmidt CM, Rosato EL, Lavu H, et al: Does type of pancreaticojejunostomy after pancreaticoduodenectomy decrease rate of pancreatic fistula? A randomized, prospective, dualinstitution trial. J Am Coll Surg 2009, 208:738-747; discussion 747739.

21. Cameron JL, Riall TS, Coleman J, Belcher KA: One thousand consecutive pancreaticoduodenectomies. Ann Surg 2006, 244:10-15.

22. Waugh JM, Clagett OT: Resection of the duodenum and head of the pancreas for carcinoma; an analysis of thirty cases. Surgery 1946, 20:224-232.

23. Akamatsu N, Sugawara Y, Komagome M, Shin N, Cho N, Ishida T, Ozawa F, Hashimoto D: Risk factors for postoperative pancreatic fistula after pancreaticoduodenectomy: the significance of the ratio of the main pancreatic duct to the pancreas body as a predictor of leakage. J Hepatobiliary Pancreat Sci 2010, 17:322-328.

24. Bartsch DK, Langer P, Kanngiesser V, Fendrich V, Dietzel K: A simple and safe anastomosis for pancreatogastrostomy using one binding purse-string and two transfixing mattress sutures. Int $J$ Surg Oncol, 2012:718637. 